\title{
ON ALMOST EINSTEIN HOLOMORPHIC VECTOR BUNDLES OVER HERMITIAN SURFACES
}

\author{
NOVICA BLAŽIIĆ
}

(Communicated by Jonathan M. Rosenberg)

\begin{abstract}
We study holomorphic vector bundles $(E, h)$ of rank 2 over a compact Hermitian surface $(M, g)$. Then the notion of a metric with a $k$ pinched Ricci curvature is introduced and it represents the generalization of the Einstein condition. Some necessary topological conditions for existence of a metric $h$ with $k$-pinched $(0 \leq k \leq 1)$ Ricci curvature are obtained.
\end{abstract}

\section{INTRODUCTION}

Let $(E, h)$ be a complex vector bundle over a 4-dimensional compact Riemmannian manifold $(M, g)$. Suppose that $E$ admits a complex connection $D$ whose Ricci curvature satisfies the Einstein condition. We may then ask what relations exist between the Chern numbers of $\mathrm{E}$.

Results of this kind are obtained in [1], [4], and [5] for the case of the tangent bundle, and in [3], [7], and [10] for the general case. In this paper, we generalize these results to the case when the Ricci curvature is "nice," that is, for example, when it is $k$-pinched. For tangent bundles, we find discussed in [11] a topological obstruction for the existence of metrics with $k$-pinched Ricci curvature.

The main result in this paper is Theorem 3.2 which generalizes the result of Lübke (see [10]). We established the inequality

$$
c_{2}(E) \geq\left\{\frac{1}{4}-\frac{(1-k)^{2}}{16 \delta}\right\} c_{1}^{2}(E)
$$

for a holomorphic vector bundle of rank 2 with $k$-pinched Ricci curvature. We also study when the equality holds in the above.

In $\S 4$ we provide some interesting examples of $k$-pinched metrics. Let $r_{1}$ and $r_{2}$ be the eigenvalues of the Ricci endomorphism of $(E, h), \operatorname{rank} E=2$. To obtain these examples we characterize the conformal classes which contain metric $h$ such that $r_{1}$ and $r_{2}$ are proportional with some constant factor $k$.

Received by the editors June 30, 1989 and, in revised form, October 23, 1989.

1980 Mathematics Subject Classification (1985 Revision). Primary 53C55, 53B35.

Key words and phrases. Holomorphic vector bundle, Ricci curvature, Chern numbers. 


\section{Preliminaries}

In this section we will follow [8, Chapter IV]. Let $(E, h)$ be a holomorphic Hermitian vector bundle of rank $\lambda$ over an Hermitian manifold $(M, g)$ of complex dimension $n$. Then $(E, h)$ admits a unique Hermitian connection $D$ and its curvature $R$ is a $(1,1)$-form with values in the bundle $\operatorname{End}(E)$. If $s=\left(s_{1}, \ldots, s_{\lambda}\right)$ is a local frame field for $E$, the curvature form $\Omega=\left(\Omega_{j}^{i}\right)$ with respect to $s$ is given by

$$
R\left(s_{j}\right)=\sum \Omega_{j}^{i} s_{i}, \quad \Omega_{j}^{i}=\sum R_{j \alpha \bar{\beta}}^{i} d z^{\alpha} \wedge d \bar{z}^{\beta}
$$

in terms of a local coordinate system $\left(z^{1}, \ldots, z^{n}\right)$ of $M$. In this paper we assume summation for every pair of repeated indexes. We write

$$
h_{i j}=h\left(s_{i}, s_{j}\right) \quad \text { and } \quad g=\sum g_{\alpha \bar{\beta}} d z^{\alpha} d \bar{z}^{\beta} .
$$

Now we define $\rho$ and $\hat{\rho}$, the Ricci and - -Ricci curvatures of $(E, h)$ respectively, by

$$
\rho_{j}^{i}=\sum g^{\alpha \bar{\beta}} R_{j \alpha \bar{\beta}}^{i}, \quad \rho_{j \bar{k}}=\sum h_{i \bar{k}} \rho_{j}^{i}
$$

and

$$
\hat{\rho}_{\alpha \bar{\beta}}=\sum h_{i}^{j} R_{j \alpha \bar{\beta}}^{i}, \quad \hat{\rho}_{\alpha}^{\beta}=\sum \hat{\rho}_{\alpha \bar{\gamma}} g^{\bar{\gamma} \beta} .
$$

Suppose now that $s=\left(s_{1}, \ldots, s_{\lambda}\right), e=\left(e_{1}, \ldots, e_{n}\right)$, and $\theta=\left(\theta^{1}, \ldots, \theta^{n}\right)$ are local unitary frame fields for $(E, h), T M$ and $T^{*} M$ respectively. Then we write

$$
\begin{aligned}
\Phi & =\sqrt{-1} \sum \theta^{\alpha} \wedge \bar{\theta}^{\alpha} \\
\|R\|^{2} & =4 \sum\left|R_{j \alpha \bar{\beta}}^{i}\right|^{2} \\
\|\rho\|^{2} & =2 \sum\left|\rho_{j}^{i}\right|^{2}=2 \sum\left|\rho_{j \bar{i}}\right|^{2} \\
\|\hat{\rho}\|^{2} & =2 \sum\left|\hat{\rho}_{\alpha \bar{\beta}}\right|^{2}=2 \sum\left|\hat{\rho}_{\alpha}^{\beta}\right|^{2} \\
\tau & =2 \sum \rho_{i}^{i}=2 \sum \hat{\rho}_{\alpha \bar{\alpha}}
\end{aligned}
$$

for the fundamental form of $(M, g)$, the norms of the tensors $R, \rho, \hat{\rho}$, and for the scalar curvature $\tau$ of $(E, h)$ respectively. Scalar curvatures and -Ricci tensors of holomorphic vector bundles $\left(E^{\prime}, h^{\prime}\right)$ and $\left(E^{\prime \prime}, h^{\prime \prime}\right)$ are denoted by $\tau^{\prime}, \tau^{\prime \prime}, \hat{\rho}^{\prime}$ and $\hat{\rho}^{\prime \prime}$ respectively. The norms of the tensors $\rho-\frac{\tau}{4} h$ and $\hat{\rho}^{\prime}-\widehat{\rho}^{\prime \prime}$ are defined by

$$
\begin{aligned}
& \left\|\rho-\frac{\tau}{4} h\right\|^{2}=2 \sum\left|\rho_{j \bar{j}}-\frac{\tau}{4} h_{j \bar{j}}\right|^{2} \\
& \left\|\hat{\rho}^{\prime}-\hat{\rho}^{\prime \prime}\right\|^{2}=2 \sum\left|\hat{\rho}_{\alpha \bar{\alpha}}^{\prime}-\hat{\rho}_{\alpha \bar{\alpha}}^{\prime \prime}\right|^{2} .
\end{aligned}
$$

From now on we suppose $\lambda=n=2$. Then let $\varrho$ and $\varrho$ denote the sections of the bundles $\operatorname{End}(E)$ and $\operatorname{End}(T M)$ defined by

$$
h(\varrho(s), t)=\rho(s, t) \quad \text { and } \quad g(\widehat{\varrho}(e), f)=\hat{\rho}(e, f)
$$


for $s, t \in E_{p}$ and $e, f \in T M_{p}, p \in M$. The endomorphisms $\varrho$ and $\varrho$ are symmetric, so their corresponding eigenvalues $r_{1}, r_{2}$ and $\widehat{r}_{1}, \widehat{r}_{2}$ are real. We will use a local unitary frame field $s=\left(s_{1}, s_{2}\right)$ determined by the eigenvectors of $\varrho$ corresponding to $r_{1}$ and $r_{2}$. Also, a local unitary frame field $e=\left(e_{1}, e_{2}\right)$ is determined by the eigenvectors of $\hat{\varrho}$.

Let $r=\max \left\{\left|r_{1}\right|,\left|r_{2}\right|\right\}$. Then, for $0 \leq k \leq 1$, we say that Ricci curvature $\rho$ of $(E, h)$ is $k$-pinched if

$$
k r h \leq \rho \leq r h \quad \text { or } \quad-r h \leq \rho \leq-k r h
$$

holds on $M$. Then, clearly, $r_{1} r_{2} \geq 0$ on $M$. When $k=1,(2.3)$ represents the weak Einstein condition as defined by Kobayashi.

The Gauss curvature $\hat{\tau}$ of $(E, h)$ can be defined by

$$
\widehat{\tau}=\operatorname{det} \widehat{\varrho}=\widehat{r}_{1} \cdot \widehat{r}_{2} \text {. }
$$

The Gauss curvature $\widehat{\tau}$ is $\delta$-bounded from below if

$$
\widehat{\tau} \geq \delta r^{2}
$$

on $M$. The class of holomorphic vector bundles which satisfy Conditions (2.3) and (2.4) is denoted by $\mathscr{E}_{k, \delta}$.

We now prove the key lemma of the paper.

Lemma 2.1. Let $(E, h)$ be a holomorphic vector bundle of rank 2 over an Hermitian surface $(M, g)$. Then the following inequality holds

$$
\|R\|^{2} \geq\|\hat{\rho}\|^{2}+\left\|\rho-\frac{\tau}{4} h\right\|^{2} \text {. }
$$

Proof. The definition of a norm of the tensor $R$ implies

$$
\|R\|^{2} \geq\|\widehat{\rho}\|^{2}+2\left(\left|R_{1 \overline{1} 1 \overline{1}}-R_{1 \overline{1} 2 \overline{2}}\right|^{2}+\left|R_{2 \overline{2} 1 \overline{1}}-R_{2 \overline{2} 2 \overline{2}}\right|^{2}\right) .
$$

Because of

$$
2\left(\left|R_{1 \overline{1} 1 \overline{1}}-R_{1 \overline{1} \overline{2} \overline{2}}\right|^{2}+\left|R_{2 \overline{2} 1 \overline{1}}-R_{2 \overline{2} 2 \overline{2}}\right|^{2}\right) \geq\left|\rho_{11}-\rho_{22}\right|^{2}
$$

and

we have $(2.5)$.

$$
\left\|\rho-\frac{\tau}{4} h\right\|^{2}=\left|\rho_{11}-\rho_{22}\right|^{2}
$$

When $(E, h)$ satisfies the weak Einstein condition, i.e. $\rho$ is a 1-pinched, the equality case was studied in [8]. It was shown that the equality holds if and only if $(E, h)$ is projectively flat, i.e. $R=\frac{1}{2} \widehat{\rho} \otimes h$. So it is natural to study when the equality holds in the general case.

Lemma 2.2. Let $(E, h)$ be a holomorphic vector bundle of rank 2 over an Hermitian surface $(M, g)$ such that the Ricci curvature tensor $\rho$ is parallel and $r_{1} \neq r_{2}$ on $M$. Then the equality

$$
\|R\|^{2}=\|\widehat{\rho}\|^{2}+\left\|\rho-\frac{\tau}{4} h\right\|^{2}
$$


holds if and only if $E$ splits

$$
E=E^{\prime} \oplus E^{\prime \prime}
$$

for some holomorphic orthogonal line bundles $\left(E^{\prime}, h^{\prime}\right),\left(E^{\prime \prime}, h^{\prime \prime}\right)$ in such a way that

$$
\hat{\rho}^{\prime}-\widehat{\rho}^{\prime \prime}=\frac{1}{4}\left(\tau^{\prime}-\tau^{\prime \prime}\right) g .
$$

Remark. If $r_{1}=r_{2}$ on $M$ the same conditions imply the equality (2.6).

Proof. Suppose that the condition (2.7) is satisfied. Then for $E=E^{\prime} \oplus E^{\prime \prime}$ we have

$$
\begin{gathered}
\|R\|^{2}=2\left(\left\|\hat{\rho}^{\prime}\right\|^{2}+\left\|\hat{\rho}^{\prime \prime}\right\|^{2}\right), \quad \hat{\rho}=\hat{\rho}^{\prime}+\widehat{\rho}^{\prime \prime}, \\
\tau^{\prime}=2 \rho_{11} \quad \text { and } \quad \tau^{\prime \prime}=2 \rho_{22} .
\end{gathered}
$$

Thus,

$$
\|R\|^{2}-\|\hat{\rho}\|^{2}-\left\|\rho-\frac{\tau}{4} h\right\|^{2}=\left\|\hat{\rho}^{\prime}-\widehat{\rho}^{\prime \prime}\right\|^{2}-\frac{1}{4}\left|\tau^{\prime}-\tau^{\prime \prime}\right|^{2}=0 .
$$

Assume now that the equality holds and $r_{1} \neq r_{2}$ on $M$. Then the subbundles $E^{\prime}$ and $E^{\prime \prime}$ of $E$ can be defined by

$$
\begin{aligned}
& E_{p}^{\prime}=\left\{t \in E_{p} \mid \varrho(t)=r_{1} t\right\} \\
& E_{p}^{\prime \prime}=\left\{t \in E_{p} \mid \varrho(t)=r_{2} t\right\}
\end{aligned}
$$

for $p \in M$. Since the Ricci curvature tensor $\rho$ is parallel, $\left(E^{\prime}, h^{\prime}\right)$ and $\left(E^{\prime \prime}, h^{\prime \prime}\right)$ are holomorphic line bundles and $E=E^{\prime} \oplus E^{\prime \prime}$ is a global decomposition of $E$ by Theorem 3.21 in [8]. The Hermitian products $h^{\prime}$ and $h^{\prime \prime}$ are determined as restrictions of $h$. Then $R=R^{\prime}+R^{\prime \prime}$ and by the first part of the proof we get

$$
\left\|\left(\hat{\rho}^{\prime}-\hat{\rho}^{\prime \prime}\right)-\frac{1}{4}\left(\tau^{\prime}-\tau^{\prime \prime}\right) g\right\|^{2}=0
$$

i.e., the Einstein-type condition (2.7) is satisfied.

For the Chern numbers $c_{1}^{2}(E)$ and $c_{2}(E)$ of $E$ the following formulas are obtained in $[8$, p. 113]

$$
c_{1}^{2}(E)=\frac{1}{8 \pi^{2}} \int_{M}\left(\tau^{2}-2\|\hat{\rho}\|^{2}\right) \Phi^{2}
$$

and

$$
c_{2}(E)=\frac{1}{16 \pi^{2}} \int_{M}\left(\tau^{2}-2\|\rho\|^{2}-2\|\hat{\rho}\|^{2}+\|R\|^{2}\right) \Phi^{2} .
$$

We will now mention some consequences of the previous results.

Corollary 2.3. Let $(E, h)$ be a holomorphic vector bundle of rank 2 over a compact Hermitian surface $(M, g)$. If the Gauss curvature of $E$ is nonnegative, then

$$
c_{1}^{2}(E) \geq 0 .
$$


Proof. The Chern number $c_{1}^{2}(E)$ can be expressed in terms of the Gauss curvature. Since $\tau=2\left(\widehat{r}_{1}+\widehat{r}_{2}\right)$ and $\|\hat{\rho}\|^{2}=2\left(\widehat{r}_{1}^{2}+\widehat{r}_{2}^{2}\right)$, we have $\tau^{2}-2\|\hat{\rho}\|^{2}=8 \widehat{r}_{1} \cdot \widehat{r}_{2}$. The Gauss curvature $\hat{\tau}$ is defined by $\hat{\tau}=\widehat{r}_{1} \cdot \widehat{r}_{2}$. Hence, $\tau^{2}-2\|\widehat{\rho}\|^{2}=8 \hat{\tau}$ and (2.8) implies

$$
c_{1}^{2}(E)=\frac{1}{\pi^{2}} \int_{M} \hat{\tau} \Phi^{2} .
$$

Now the result follows immediately.

Remark. This result is already known (see [7, Theorem 4.1]) because $\widehat{\tau}$ is nonnegative if and only if ${ }^{-}$-Ricci curvature is nonnegative or nonpositive.

Corollary 2.4. Let $(E, h)$ be a holomorphic line bundle over a compact Hermitian surface $M$ and let $E^{*}$ be the dual bundle of $E$. Then the holomorphic bundle $E \oplus E^{*}$ does not admit metrics whose Gauss curvature is positive (or negative) on $M$.

\section{Chern Numbers $c_{1}^{2}(E)$ AND $c_{2}(E)$}

Let $e(E)$ denote the Euler characteristic of complex vector bundle $E$.

Theorem 3.1. Let $(E, h)$ be a holomorphic vector bundle of rank 2 over a compact Hermitian surface $(M, g)$. If the Ricci tensor is k-pinched and the Gauss curvature is $\frac{1}{4}(1-k)^{2}$-bounded from below we have

$$
e(E)=c_{2}(E) \geq 0 \text {. }
$$

Here, $e(E)$ is the Euler characteristic of $E$. If the Ricci curvature $\rho$ is parallel and $k<1$, the equality holds if and only if $(E, h)$ admits a holomorphic orthogonal decomposition $(E, h)=\left(E^{\prime}, h^{\prime}\right) \oplus\left(E^{\prime \prime}, h^{\prime \prime}\right)$ with $\widehat{\rho}^{\prime}-\widehat{\rho}^{\prime \prime}=\frac{1}{4}(1-k) r g$ and $\hat{\tau}=\frac{1}{4}(1-k)^{2} r^{2}$. For $k=1$ the same conditions imply the equality.

Proof. Using the formulas (2.8) and (2.9) and Lemma 2.1. we have

$$
\begin{aligned}
e(E)=c_{2}(E) & =\frac{1}{16 \pi^{2}} \int_{M}\left(\tau^{2}-2\|\rho\|^{2}-2\|\hat{\rho}\|^{2}+\|R\|^{2}\right) \Phi^{2} \\
& \geq \frac{1}{16 \pi^{2}} \int_{M}\left\{4 \hat{\tau}-\left(\rho_{11}-\rho_{22}\right)^{2}\right\} \Phi^{2} .
\end{aligned}
$$

Since $\rho$ is $k$-pinched and $\widehat{\tau}$ is $\frac{1}{4}(1-k)^{2}$-bounded we get $e(E) \geq 0$.

Assume that the equality holds. Then the equality holds in $(2.5)$ and $\widehat{\tau}=$ $\frac{1}{4}(1-k)^{2} r^{2}$ and $\left|\rho_{11}-\rho_{22}\right|^{2}=(1-k)^{2} r^{2}$. Thus $\widehat{\tau}^{\prime}-\widehat{\tau}^{\prime \prime}=(1-k) r$. Then by Lemma 2.2. we complete proof of Theorem 3.1.

For an Einstein-Hermitian vector bundle $(E, h)$, Lübke [10] (cf. also [8]) established the following inequality

$$
c_{2}(E) \geq \frac{1}{4} c_{1}^{2}(E)
$$

The equality holds in (3.1) if and only if $(E, h)$ is a projectively flat vector bundle. 
In our next theorem, we generalize the inequality (3.1) for bundles in the class $\mathscr{E}_{k, \delta}$.

Theorem 3.2. Let $(E, h)$ be an Hermitian vector bundle of rank 2 over a compact Hermitian surface $(M, g)$ which belongs to $\mathscr{C}_{k, \delta}, \delta \geq 0$. Then

$$
c_{2}(E) \geq\left\{\frac{1}{4}-\frac{(1-k)^{2}}{16 \delta}\right\} c_{1}^{2}(E) .
$$

If the Ricci curvature $\rho$ is parallel and $k<1$, the equality holds if and only if $(E, h)$ admits a holomorphic orthogonal decomposition

$$
(E, h)=\left(E^{\prime}, h^{\prime}\right) \oplus\left(E^{\prime \prime}, h^{\prime \prime}\right)
$$

with $\hat{\rho}^{\prime}-\widehat{\rho}^{\prime \prime}=\frac{1}{4}(1-k) r g$ and $\hat{\tau}=\delta r^{2}$.

Remark. In the Einstein-Hermitian case, i.e. for $\mathrm{k}=1$, the inequality (3.2) is reduced to the inequality (3.1). The equality holds if $(E, h)$ admits decomposition (3.3) with $\hat{\rho}^{\prime}=\hat{\rho}^{\prime \prime}$ (see [10]).

Proof. Using (2.8), (2.9), and Lemma 2.1 we get

$$
16 \pi^{2}\left(c_{2}(E)-a c_{1}^{2}(E)\right) \geq \int_{M}\left\{4(1-4 a) \widehat{\tau}-\left\|\rho-\frac{\tau}{4} h\right\|^{2}\right\} \Phi^{2}
$$

for any real number $a$. Then $\tau=2\left(r_{1}+r_{2}\right)$ and

$$
\left\|\rho-\frac{\tau}{4} h\right\|^{2}=2\left(\left|r_{1}-\frac{\tau}{4}\right|^{2}+\left|r_{2}-\frac{\tau}{4}\right|^{2}=\left|r_{1}-r_{2}\right|^{2} .\right.
$$

When the Ricci curvature $\rho$ is $k$-pinched, we easily see that (3.2) implies

$$
\left|r_{1}-r_{2}\right| \leq(1-k) r \text {. }
$$

Thus, for $(E, h) \in \mathscr{E}_{k, \delta}$ we have

$$
\widehat{\tau} \geq \delta r^{2} \quad \text { and } \quad\left\|\rho-\frac{\tau}{4} h\right\|^{2} \leq(1-k)^{2} r^{2} .
$$

Moreover, (3.4) and (3.5) imply

$$
4(1-4 a) \hat{\tau}-\left\|\rho-\frac{\tau}{4} h\right\|^{2} \geq\left\{4(1-4 a) \delta-(1-k)^{2}\right\} r^{2} .
$$

Combining (3.4) and (3.6) we obtain

$$
16 \pi^{2}\left(c_{2}(E)-a c_{1}^{2}(E)\right) \geq \int_{M}\left\{4(1-4 a) \delta-(1-k)^{2}\right\} r^{2} \Phi^{2} .
$$

Notice that $4(1-4 a) \delta-(1-k)^{2}$ vanishes for $a=\frac{1}{4}-(1-k)^{2} / 16 \delta$. Thus, substituting $a=\frac{1}{4}-(1-k)^{2} / 16 \delta$ in (3.7) we obtain the inequality (3.2).

Now, assume that the equality holds in (3.2). Then the equality holds in (2.5) and

$$
\widehat{\tau}=\delta r^{2} \quad \text { and } \quad\left|\rho_{11}-\rho_{22}\right|^{2}=(1-k)^{2} r^{2} .
$$

Thus $\hat{\tau}^{\prime}-\hat{\tau}^{\prime \prime}=(1-k) r$. Then by Lemma 2.2, we complete proof of Theorem 3.2 . 
Remark. The inequalities (2.5) and (3.2) also hold for formally holomorphic vector bundles.

\section{Conformal Change of BUNDle Metric}

Let $(E, h)$ be a holomorphic Hermitian vector bundle of rank 2 over a compact Kähler manifold $(M, g)$ of complex dimension $\mathrm{n}$. We choose $k$ to be the constant such that

$$
\int_{M} r_{1} \Phi^{n}=k \int_{M} r_{2} \Phi^{n}
$$

Theorem 4.1. Let $(E, h)$ be an Hermitian vector bundle of rank 2 over a compact Kähler manifold $(M, g)$ and let $k$ be the constant defined by (4.1). Then, if $k \neq 1$, there is a conformally equivalent Hermitian structure $h^{\prime}=a h, a$ is a real positive function on $M$, such that

$$
r_{1}^{\prime}=k r_{2}^{\prime}
$$

on $M$. This metric is unique up to homothety.

Proof. Let $a$ be a real, positive function on $M$ and let $h^{\prime}=a h$ be a new Hermitian structure with the Ricci curvature tensor $\rho_{j \bar{k}}^{\prime}$ and the corresponding endomorphism $\varrho^{\prime}$. Then

$$
\rho_{j \bar{k}}^{\prime}=a \rho_{j \bar{k}}-(\triangle \log a) a h_{j \bar{k}} ;
$$

where $\triangle=g^{\alpha \bar{\beta}} \partial_{\alpha} \partial_{\bar{\beta}}$. Because of (4.3) we have

$$
r_{1}^{\prime}=r_{1}-\triangle \log a \quad \text { and } \quad r_{2}^{\prime}=r_{2}-\triangle \log a,
$$

where $r_{1}, r_{2}$ and $r_{1}^{\prime}, r_{2}^{\prime}$ are the eigenvalues of $\varrho$ and $\varrho^{\prime}$ respectively. Moreover, $r_{1}-r_{2}$ is a conformal invariant.

Then, the differential equation (4.2) can be written as

$$
r_{1}-k r_{2}=(1-k)(\triangle \log a)
$$

or

$$
\triangle \log a=f
$$

where $f=\left(r_{1}-k r_{2}\right) /(1-k)$.

Now, we will establish the existence of a globally defined solution of (4.4). Since $\Delta$ is a self-adjoint elliptic operator of order 2 , we can apply Hodge's decomposition theorem (see [6], p. 42) which says:

$$
C^{\infty}(M)=\operatorname{Ker}(\Delta) \oplus \Delta\left(C^{\infty}(M)\right) .
$$

So, there are the harmonic function $f_{0}$ and the smooth function $f_{1}$ on $M$ such that

$$
f=f_{0}+\triangle f_{1}
$$


The decomposition (4.5) is orthogonal, i.e., $\operatorname{Ker}(\triangle) \perp \triangle\left(C^{\infty}(M)\right)$, hence we have

$$
\int_{M} \triangle f_{1} \Phi^{n}=0
$$

because constant functions lie in $\operatorname{Ker}(\triangle)$. From (4.1) directly follows $\int f \Phi^{n}$ $=0$. Now, using (4.6) we show

$$
\int_{M} f_{0} \Phi^{n}=0
$$

Then, because $M$ is compact, we can apply Hopf lemma to see that $f_{0}$ is constant on $M$. So, (4.7) implies $f_{0} \equiv 0$ on $M$. Then, $a=\exp \left(f_{1}\right)$ is the global solution of the equation (4.4).

It is known that the kernel of the Laplacian $\triangle$ on a compact manifold consists of constant functions. Hence, this implies the uniqueness property and the proof is completed.

Remark. From the given proof follows that $r_{1}-r_{2}$ is a conformal invariant.

Remark. For $k=1$, Theorem 4.1 holds if and only if metric $h$ is an Einstein metric.

Corollary 4.2. Under the same assumptions as in Theorem 4.1, if $\int r_{1} \Phi^{n} \cdot \int r_{2} \Phi^{n}$ $\geq 0$ and $\left|\int r_{1} \Phi^{n}\right|<\left|\int r_{2} \Phi^{n}\right|,(E, h)$ has $k$-pinched Ricci curvature with a constant $k$ defined by the formula (4.1).

\section{ACKNOWLEDGMENTS}

Many thanks are due to the referee for number of useful corrections and improvements in the exposition. I also wish to thank Neda Bokan for helpful discussions.

\section{REFERENCES}

1. M. Berger, Sur les variétés d'Einstein compactes, C. R. IIIe Reunion Math. Expression latine, Namur (1965), 35-55.

2. A. Besse, Seminaire Arthur Besse 1978/79, Cedic/Fernand Nathan, Paris, 1981.

3. N. Blažić, Chern classes of complex vector bundles over almost complex manifolds, Boll. Un. Mat. Ital. B 7 (1989).

4. B.-Y. Chen and K. Ogiue, Some characterizations of complex space forms in terms of Chern classes, Quart. J. Math. Oxford 26 (1975), 459-464.

5. P. Gauduchon, La topologie d'une surface hermitienne d'Einstein, C. R. Acad. Sci. Paris Sér. A 290 (1980), 509-512.

6. P. Gilkey, The index theorem and the heat equation, Publish or Perish, 1974.

7. A. Gray, M. Baros, A. Naveira, and L. Vanhecke, The Chern numbers of holomorphic vector bundles and formally holomorphic connections of complex vector bundles over almost complex manifolds, J. Reine Angew. Math. 8 (1980), 84-98.

8. S. Kobayashi, Differential geometry of complex vector bundles, Princeton University Press, Princeton, 1987.

9. _ Curvature and stability of vector bundles, Proc. Japan Acad. Ser. A 58 (1982), $158-162$. 
10. M. Lübke, Chernklassen von Hermite-Einstein-vectorbündeln, Math. Ann. 260 (1982), 133141.

11. A. Polombo, Nombres caracteristiques d'une variete Riemannienne de dimension 4, J. Differential Geom. 13 (1978), 145-162.

Faculty of Mathematics, University of Belgrade, Studentski trg 16, P.P. 550, 11000 BEOGRAD, YugosLaVia 